Araştırma Makalesi-Research Article

\title{
Düz Disklerdeki Bilardo Akışlarının Topolojik Geçişliliği
}

\author{
İsmail Sağlam ${ }^{1 *}$, Ahmet Refah Torun ${ }^{2}$ \\ Geliş / Received: 09/07/2019 \\ Revize / Revised: 28/02/2020 \\ Kabul / Accepted: 03/03/2020 \\ ÖZ \\ Sabit bir iç açı datasına sahip her düz diskler ailesinin bilardo akışı topolojik geçişli olan yoğun bir $\mathrm{G}_{\delta}$ kümesi içerdiğini \\ gösteriyoruz.
}

Anahtar Kelimeler- Topolojik geçişlilik, Düz disk, Bilardo akışı

1*Sorumlu yazar iletișim: isaglam@atu.edu.tr (https://orcid.org/0000-0002-1238-6396)

Havacılık ve Uzay Mühendisliği Bölümü, Adana Alparslan Türkeş Bilim ve Teknoloji Üniversitesi, Adana/Türkiye 2İletişim: artorun@atu.edu.tr (https://orcid.org/0000-0001-7213-5228)

Havacılık ve Uzay Mühendisliği Bölümü, Adana Alparslan Türkeş Bilim ve Teknoloji Üniversitesi, Adana/Türkiye 


\title{
Topological Transitivity of Billiard Flows in Flat Disks
}

\author{
ABSTRACT \\ We show that any space of flat disks having a fixed integral interior angle data and a fixed number of singular points \\ contains a dense $\mathrm{G}_{\delta}$ set so that for each element of this set billiard flow is topologically transitive.
}

Keywords- Topological transitivity, Flat disk, Billiard flow 


\section{INTRODUCTION}

Dynamics of billiard flows on polygonal billiard tables is a highly active research area. Ergodicity and minimality of billiard flows, existence of periodic orbits of billiard flows, number of periodic orbits, topological transitivity of billiard flows are among the topics which are studied in the area [1-5].

A flat disk is a topological disk with a flat metric on it. Polygons are examples of flat disks with nonsingular interiors. It is curious that which properties of billiard flows on polygons are valid for flat disks. It is known that space of polygons with $n$ vertices has a $G_{\delta}$ dense set consisting of polygons with topologically transitive billiard flows. Now fix an angle data $\Theta=\left(2 \pi k_{l}, \ldots, 2 \pi k_{n}\right)$ and an integer $m \geq 0$, where each $k_{i}$ is a natural number greater than or equal to 2 . Consider the space of flat disks whose singular interior points have angle data $\Theta$ and which has $m$ singular boundary points. In this work, we prove that this space has a dense $G_{\delta}$ set so that each element of the set has topologically transitive billiard flow.

It is easy to parametrize spaces of polygons since each polygon is a subset of the Euclidean plane. However it is not straight forward to identify the spaces of certain flat metrics on a surface with a manifold. We start by stating a result about moduli space of flat metrics on the sphere with a prescribed angle data. Then we use this result to parametrize moduli space of flat disks with prescribed curvature data. After that, we obtain a manifold structure for the space of flat metrics with prescribed interior angle data.

A closed surface is called really flat if it has a finite holonomy group, and it is very flat if it has trivial holonomy group. We state a theorem proven in [6] which says that any really flat surface can be covered by a very flat surface. We then prove the main result. Two proofs of this statement for the case of the polygons are given in $[5,7]$. Our proof is similar to them.

\section{FLAT SURFACES AND FLAT DISKS}

In this section we give some generalities about flat surfaces and introduce the families of the flat disks that we consider. We define flatness for only compact surfaces since we are interested only in these surfaces.

\section{Flat Surfaces and Gauss-Bonnet Formula}

A compact surface is flat if it has a metric obtained by gluing finitely many triangles along its edges by Euclidean isometries. See [6-11] for more information about flat surfaces. Note that we can talk about the angle at a point of a flat surface. If $S$ is flat surface and $p \in S$, we denote the angle at $p$ by $\theta(p)$. If $p$ is interior point and angle at $p$ is not $2 \pi$, we call $p$ singular. If $p$ is a boundary point and angle at $p$ is not $\pi$, we call it singular. A point which is not singular is called non-singular. Let int $(S)$ be the interior of $S$ and $b(S)$ be the boundary of $S$. Here is the famous Gauss-Bonnet Formula:

$$
\sum_{p \in \text { int }(S)}(2 \pi-\theta(p))+\sum_{p \in b(S)}(\pi-\theta(p))=2 \pi \chi(S)
$$

where $\chi(S)$ is the Euler characteristics of $S$.

\section{Flat Disks}

A flat disk is an oriented topological disk together with a flat metric on it. We assume that singular points of a flat disk are labeled. Two flat metrics are equivalent if there is an orientation preserving isometry between some representatives of their homothety classes such that this isometry respects the labellings. Let $\Theta=\left(\theta_{1}, \ldots, \theta_{n}\right)$ be a finite sequence of positive numbers so that each $\theta_{i} \neq 2 \pi$. We call $\Theta$ interior angle data. Let $m \geq 0$ be an integer. Let $D(\Theta, m)$ denote set of equivalence classes of flat disks with $m$ labeled singular 
boundary points and $n$ labeled interior points having angles $\theta_{1}, \ldots, \theta_{n}$. We know that the space of the flat disks with prescribed angle data is nonempty if the data satisfies Gauss-Bonnet condition for disks (1). From this we see that $D(\Theta, m)$ is nonempty if and only if there exists positive real numbers $\alpha_{1}, \ldots, \alpha_{m} \neq \pi$ such that

$$
\sum_{i=1}^{n}\left(2 \pi-\theta_{i}\right)+\sum_{i=1}^{m}\left(\pi-\alpha_{i}\right)=2 \pi .
$$

Therefore $D(\Theta, m)$ is nonempty if and only if

1. $\sum_{i=1}^{n}\left(2 \pi-\theta_{i}\right)=2 \pi$ and $m=0,2,3,4, \ldots$,

2. $\sum_{i=1}^{n}\left(2 \pi-\theta_{i}\right)>2 \pi$ and $m>0$, or

3. $\sum_{i=1}^{n}\left(2 \pi-\theta_{i}\right)<2 \pi$ then $m \geq 3+\left\lfloor\frac{\sum_{i=1}^{n}\left(\theta_{i}-2 \pi\right)}{\pi}\right\rfloor$

From now on we assume $D(\Theta, m)$ is not empty. Now we introduce another type of families of flat diks. Fix an interior angle data $\Theta$ and let $A=\left(\alpha_{1}, \ldots, \alpha_{m}\right)$ such that each $\alpha_{i} \neq \pi, \alpha_{1} \geq \alpha_{2} \geq \cdots \geq \alpha_{m}>0$ and $(\Theta, A)$ satisfy Gauss-Bonnet condition 1 . We call such a pair $(\Theta, A)$ a good pair. Now let $D(\Theta, A)$ be the set of equivalence classes of labeled flat disks having angle data $(\Theta, A)$, where two flat metrics are equivalent if there is an orientation preserving isometry between some representatives of their homothety classes such that this isometry respects the labellings.

Let $E(\Theta)$ be the set of tuples $A=\left(\alpha_{1}, \ldots, \alpha_{m}\right)$ such that $(\Theta, A)$ is a good pair.

$$
D(\Theta, m)=\mathrm{U}_{A \in E(\Theta)} D(\Theta, A)
$$

We know that $D(\Theta, A)$ is not empty if $A \in E(\Theta)$. See [10] Thus $D(\Theta, m)$ is not empty exactly when $E(\Theta)$ is not empty.

\section{Moduli space of points on complex projective line and upper half-plane}

Our aim is to endow the families of flat metrics constructed above with differential structures. To do this, we introduce some of the manifolds that we need. Let

1. $\widehat{\mathbb{C}}=\mathbb{C} \cup\{\infty\}$ be the Riemann sphere,

2. $\mathbb{H}=\{x+\imath y \mid x, y \in \mathbb{R}, y>0\}$ be the upper half-plane,

3. $\overline{\mathbb{H}}=\{x+\imath y \mid x, y \in \mathbb{R}, y \geq 0\} \cup\{\infty\}$,

4. $\widehat{\mathbb{R}}=\mathbb{R} \cup \infty$,

5. $\widehat{\mathbb{C}}(n)=\left\{\left(z_{1}, \ldots, z_{n}\right) \mid z_{i} \in \widehat{\mathbb{C}}, z_{i} \neq z_{j}\right.$ if $\left.i \neq j\right\}$, $x \_\mathrm{i} \_\mathrm{j}$ if $\left.\mathrm{i} \mathrm{j},\right\}$

6. $\overline{\mathbb{H}}(n, m)=\left\{(\hat{z}, \hat{x}) \mid @ l @ \mathrm{z}=\left(\mathrm{z} \_1, \mathrm{z} \_2, \mathrm{z} \_\mathrm{n}\right), \mathrm{z} \_\mathrm{i} \mathrm{H}, \mathrm{z} \_\mathrm{i} \mathrm{z} \_\mathrm{j}\right.$ if $\mathrm{i} \mathrm{j}, \mathrm{x}=\left(\mathrm{x} \_1, \mathrm{x} \_2, \mathrm{x} \_\mathrm{m}\right), \mathrm{x} \_\mathrm{i} \quad \mathrm{R}$, 
7. $M(\widehat{\mathbb{C}}, n)=\widehat{\mathbb{C}}(n) / P G L(2, \mathbb{C})$,

8. $M(\overline{\mathbb{H}}, n, m)=\overline{\mathbb{H}}(n, m) / \operatorname{PSL}(2, \mathbb{R})$.

$\overline{\mathbb{H}}(n, m)$ has dimension $2 n+m$, and dimension of $\operatorname{PSL}(2, \mathbb{R})$ is 3 . Therefore, if $2 n+m \geq 3$, then the dimension of $M(\overline{\mathbb{H}}, n, m)$ is $2 n+m-3$. Otherwise $M(\overline{\mathbb{H}}, n, m)$ is a finite set. The complex dimension of $M(\mathbb{C}, n)$ is $n-3$ if $n \geq 3$. Otherwise $M(\mathbb{C}, n)$ is one point set.

\section{Flat Spheres and their moduli space}

Assume that $\theta_{1}, \ldots, \theta_{n}$ are positive real numbers which are not equal to $2 \pi$ and satisfy Gauss-Bonnet condition for the sphere:

$$
\sum_{i=1}^{n}\left(2 \pi-\theta_{i}\right)=4 \pi
$$

Let $\theta=\left(\theta_{1}, \ldots, \theta_{n}\right)$. Let $S$ and $S^{\prime}$ be two oriented topological spheres with flat metrics having angle data $\theta$. We say that $S$ and $S^{\prime}$ are equivalent if there are two isometric flat spheres in their homothety classes. Note that the isometries that we consider respect labeling and they are orientation preserving. We denote the set of equivalence classes of the flat spheres with angle data $\theta$ by $C\left(S^{2}, \theta\right)$.

$$
\begin{aligned}
& \text { Let } \beta_{i}=\frac{\theta_{i}}{2 \pi}-1 \text {. Then the metric given } \\
& d s^{2}=\prod_{i=1}^{n}\left|z-z_{i}\right|^{2 \beta_{i}}|d z|^{2}
\end{aligned}
$$

is defined on $\widehat{\mathbb{C}}$ and flat, where $\left(z_{1}, \ldots, z_{n}\right) \in \widehat{\mathbb{C}}(n)$. Also the angle at each $z_{i}$ is $\theta_{i}$ and the other points are non-singular. See [9] This means that there is a map

$$
m: \widehat{\mathbb{C}}(n) \rightarrow C\left(S^{2}, \theta\right) .
$$

This map is surjective but not injective. Indeed,

$$
\widehat{\mathbb{C}}(n) / P G L(2, \mathbb{C}) \equiv C\left(S^{2}, \theta\right) .
$$

See [8],[9]. We summarize the results of this section in the following lemma.

Lemma 1 The map $m: \widehat{\mathbb{C}}(n) \rightarrow C\left(S^{2}, \theta\right)$ sending $\hat{z}=\left(z_{1}, \ldots, z_{n}\right)$ to the metric on $\widehat{\mathbb{C}}$ given by

$$
d s^{2}=\prod_{i=1}^{n}\left|z-z_{i}\right|^{2 \beta_{i}}|d z|^{2},
$$

is surjective. Moreover, $\hat{z}=\left(z_{1}, \ldots, z_{n}\right)$ and $\hat{z}^{\prime}=\left(z_{1}^{\prime}, \ldots, z_{n}^{\prime}\right)$ have the same image under $m$ if and only if there is an element $g$ in $P G L(2, \mathbb{C})$ such that $g \hat{z}=\hat{z}^{\prime}$. That is,

$$
M(\widehat{\mathbb{C}}, n) \equiv \widehat{\mathbb{C}}(n) / P G L(2, \mathbb{C}) \equiv C\left(S^{2}, \theta\right) .
$$

Also, the map $g: \widehat{\mathbb{C}} \rightarrow \widehat{\mathbb{C}}$ is an isometry between the flat structures which correspond to $\hat{z}$ and $\hat{z}^{\prime}$ 
A note: Indeed, if one of the coordinates $z_{j}$ of $\hat{z}=\left(z_{1}, \ldots, z_{n}\right)$ is $\infty$, then the metric in (4) does not make sense. But in that case we may consider the following metric:

$$
d s^{2}=\prod_{i=1, i \neq j}^{n}\left|z-z_{i}\right|^{2 \beta_{i}}|d z|^{2} .
$$

Flat disks and their moduli spaces

Let $\Theta=\left(\theta_{1}, \ldots, \theta_{m}\right)$ and $A=\left(\alpha_{1}, \ldots, \alpha_{m}\right)$ so that $(\Theta, A)$ is a good pair.

Proposition 1 The map $m^{\prime}: \overline{\mathbb{H}}(n, m) \rightarrow D(\Theta, A)$ sending $\left(z_{1}, \ldots z_{n}, x_{1}, \ldots, x_{m}\right)$ to the flat disk $\overline{\mathbb{H}}$ together with the metric

$$
d s^{2}=\prod_{i=1}^{n}\left|z-z_{i}\right|^{2 \beta_{i}} \prod_{i=1}^{n}\left|z-\bar{z}_{i}\right|^{2 \beta_{i}} \prod_{i=1}^{m}\left|z-x_{i}\right|^{2 \beta r_{i}}|d z|^{2},
$$

is surjective, where $\beta_{i}=\frac{\theta_{i}}{2 \pi}-1, \quad \beta_{i}^{\prime}=\frac{2 a_{i}}{2 \pi}-1$. Moreover, $(\hat{z}, \hat{x})=\left(z_{1}, \ldots, z_{n}, x_{1}, \ldots, x_{m}\right)$ and $\left(\hat{z}^{\prime}, \hat{x}^{\prime}\right)=\left(z_{1}^{\prime}, \ldots, z_{n}^{\prime}, x_{1}^{\prime}, \ldots, x_{m}^{\prime}\right)$ have the same image under $m^{\prime}$ if and only if there is an element $g$ in $\operatorname{PSL}(2, \mathbb{R})$ such that $g(\hat{z}, \hat{x})=\left(\hat{z}^{\prime}, \hat{x}^{\prime}\right)$. That is,

$$
M(\overline{\mathbb{H}}, n, m)=\overline{\mathbb{H}}(n, m) / P S L(2, \mathbb{R}) \equiv D(\Theta, A)
$$

Proof. Let $D$ be a representative for an element in $D(\Theta, A)$. Glue two copies of $D$ along their boundaries to get a flat sphere. This sphere is called the doubling of $D$, and we denote it by $d(D)$. It has $2 n$ singular points of angles $\theta_{1}, \ldots, \theta_{n}, \theta_{1}, \ldots, \theta_{n}$ and $m$ singular points of angles $\alpha_{1}, \ldots, \alpha_{m}$. Then, by Lemma 1 , there is an element $\left(z_{1}, z_{2}, \ldots, z_{n}, z_{1}^{\prime}, \ldots, z_{n}^{\prime}, z^{\prime \prime}{ }_{1}, \ldots, z^{\prime \prime}{ }_{m}\right)$ in $\widehat{\mathbb{C}}(2 n+m)$ such that $D$ is isometric to $\widehat{\mathbb{C}}$, perhaps after scaling, where $\widehat{\mathbb{C}}$ has the following metric:

$$
\prod_{i=1}^{n}\left|z-z_{i}\right|^{2 \beta_{i}} \prod_{i=1}^{n}\left|z-z_{i}^{\prime}\right|^{2 \beta_{i}} \prod_{i=1}^{m}\left|z-z^{\prime \prime}{ }_{i}\right|^{2 \beta r_{i}}|d z|^{2},
$$

where $\beta_{i}=\frac{\theta_{i}}{2 \pi}-1, \beta_{i}^{\prime}=\frac{2 \alpha_{i}}{2 \pi}-1$.

The reflection on $d(S)$ corresponds to an anti-holomorphic map of the form $f(z)=\frac{a \bar{z}+b}{c \bar{z}+d}$, and it fixes a circle $c$ in $\widehat{\mathbb{C}}$ such that $z^{\prime \prime}{ }_{1}, \ldots, z^{\prime \prime}{ }_{m} \in c$ and $f\left(z_{i}\right)=z_{i}^{\prime}$ for all $1 \leq i \leq n$. Let $h$ be a Mobius transformation sending $c$ to $\widehat{\mathbb{R}}$. By Lemma $1, \widehat{\mathbb{C}}$ together with the metric

$$
\prod_{i=1}^{n}\left|z-h\left(z_{i}\right)\right|^{2 \beta_{i}} \prod_{i=1}^{n}\left|z-h\left(z_{i}^{\prime}\right)\right|^{2 \beta_{i}} \prod_{i=1}^{m}\left|z-h\left(z^{\prime \prime}{ }_{i}\right)\right|^{2 \beta r_{i}}|d z|^{2},
$$

is isometric to $d(D)$, perhaps after scaling. The reflection of $d(D)$ corresponds to the map $h \circ f \circ h^{-1}$. But $h \circ f \circ h^{-1}$ fixes $\widehat{\mathbb{R}}$ pointwise and therefore it is equal to the map $z \rightarrow \bar{z}$. This shows that restriction of( 6) to $\overline{\mathbb{H}}$ is the metric on $D$. Now

$$
h \circ f \circ h^{-1}\left(h\left(z_{i}\right)\right)=h \circ f\left(z_{i}\right)=h\left(z_{i}^{\prime}\right),
$$

that is $\overline{h\left(z_{i}\right)}=h\left(z_{i}^{\prime}\right)$ for all $1 \leq i \leq n$. Also

$$
h \circ f \circ h^{-1}\left(h\left(z^{\prime \prime}{ }_{i}\right)\right)=h \circ f\left(z^{\prime \prime}{ }_{i}\right)=h\left(z^{\prime \prime}{ }_{i}\right),
$$


therefore $\overline{h\left(z^{\prime \prime}\right)}=h\left(z^{\prime \prime}{ }_{i}\right)$ for all $1 \leq i \leq m$. Therefore $h\left(z^{\prime \prime}{ }_{i}\right) \in \widehat{\mathbb{R}}$. This proves that $m^{\prime}$ is surjective.

Now assume that the flat structures on $\overline{\mathbb{H}}$ corresponding to

$(\hat{z}, \hat{x})=\left(z_{1}, \ldots, z_{n}, x_{1}, \ldots, x_{m}\right)$ and $\left(\hat{z}^{\prime}, \hat{x}^{\prime}\right)=\left(z_{1}^{\prime}, \ldots, z_{n}^{\prime}, x_{1}^{\prime}, \ldots, x_{m}^{\prime}\right)$ are isometric. Each metric can be naturally extended to a metric on $\widehat{\mathbb{C}}$ and the isometry extends to an isometry of $\widehat{\mathbb{C}}$. Therefore it is given by an element of $\operatorname{PGL}(2, \mathbb{C})$ fixing upper half-plane. This element is necessarily in $\operatorname{PSL}(2, \mathbb{R})$ and sends $(\hat{z}, \hat{x})$ to $\left(\hat{z}^{\prime}, \hat{x}^{\prime}\right)$.

Now fix $\Theta$. Since $D(\Theta, m)=\mathrm{U}_{A \in E(\Theta)} D(\Theta, A)$, we have

Corollary $1 D(\Theta) \equiv E(\Theta) \times M(\overline{\mathbb{H}}, n, m)$.

An interior angle data $\Theta=\left(\theta_{1}, \ldots \theta_{n}\right)$ is called integral if each $\theta_{i}=2 \pi k_{i}$, where $k_{i}$ is an integer greater than or equal to 2 . In that case, if $D(\Theta, m)$ is not empty, then $m \geq 3$. From now on we assume $\Theta$ is an integral interior angle data. For each good pair $(\Theta, A)$, we identify $D(\Theta, A)$ with the set

$$
X(n, m)=\left\{\left(z_{1}, z_{2}, \ldots, z_{n}, x_{1}, x_{2}, x_{3}, \ldots x_{m}\right) \in \overline{\mathbb{H}}(n, m): x_{1}=0, x_{2}=1, x_{3}=\infty\right\}
$$

For each element $(\hat{z}, \hat{x})=\left(z_{1}, z_{2}, \ldots, z_{n}, x_{1}, x_{2}, x_{3}, \ldots x_{m}\right) \in X(n, m)$ we have a metric induced by the metric (5) on $\overline{\mathbb{H}}$. We denote this metric by

$$
\mu(\hat{z}, \hat{x})=\mu(A,(\hat{z}, \hat{x})) .
$$

These metrics form a complete set of representatives of the flat metrics on disk with angle data $(\Theta, A)$. Therefore from now on we should consider the set $X(n, m)$ if $D(\Theta, A)$ is mentioned. We also identify $D(\Theta, m)$ with the manifold

$$
E(\Theta) \times X(n, m)
$$

\section{FLAT SURFACES WITH FINITE HOLONOMY GROUPS}

We will state the results obtained in [12].

\section{Definition 1}

1. A closed orientable flat surface is called really flat if it has finite holonomy group.

2. An orientable, compact, flat surface with boundary is called really flat if its doubling is really flat.

3. A non-orientable surface is really flat if the corresponding orientable double cover is really flat.

4. A closed, orientable, flat surface is called very flat if it has trivial holonomy group.

Thus really flat surfaces are somewhere in between flat surfaces and very flat surfaces. Let $S$ be a closed, orientable really flat surface.

\section{Theorem 1}

1. There exists a very flat surface $S^{*}$ and a branched covering $\psi: S^{*} \rightarrow S$ such that $\psi$ respects flat metrics on $S$ and $S^{*}$. Also this map corresponds to the kernel of the holonomy representation of $S$.

2. $\psi$ is a cyclic Galois covering. 

in the fiber of $x$ is $l$.

3. If $x \in S$ has an angle $\frac{2 \pi k}{l}, \mathrm{k}, 1$ are coprime natural numbers, then ramification index at each point

4. The degree of $\psi$ is the order of the holonomy group.

We call $\psi$ canonical covering. Now let $D$ be a rational flat sphere with. Let $\left\{x_{1}, \ldots, x_{n}\right\}$ be the singular interior points and $\left\{y_{1}, \ldots y_{m}\right\}$ be the set of singular boundary points of $D$. Assume that for each $i$ the angle at $x_{i}$ is of the form $\frac{2 \pi k_{i}}{l_{i}}$, and the angle at each $y_{j}$ is of the form $\frac{\pi r_{j}}{l_{j}}$, where $k_{i}$ and $l_{i}$ are coprime natural numbers, and so are $k_{j}^{\prime}$ and $l_{j}^{\prime}$. Let $\Pi: d(D) \rightarrow D$ be the canonical projection and $\psi: d(D)^{*} \rightarrow d(D)$ be the canonical covering of $d(D)$.

Theorem 2 Let $l$ be that least common multiple of $l_{1}, \ldots, l_{n}, l_{1}^{\prime}, \ldots, l_{m}^{\prime}$.

1. Then degree of $\psi$ is $l$.

2. The ramification index of each point in the fiber of $x_{i}$, or its mirror symmetry, is $l_{i}$. The ramification index of each point in the fiber of $y_{j}$ is is $l_{j}^{\prime}$.

Indeed, if the interior of $D$ is non-singular, then $d(D)$ is the surface constructed by Katok-Zemlyakov [11] which is associated to $D$.

\section{DYNAMICS ON FLAT DISKS}

Let $D$ be a flat disk. The billiard flow we consider is the flow obtained by frictionless motion of a point particle inside $D$ which obeys the rule of optics on the boundary: the angle of incidence is equal to the angle of reflection. This means that collisions are elastic. The phase space of the flow is unit tangent bundle $U(D)$. The billiard flow has discontinuities corresponding to reflections in the boundary.

Let $\Theta=\left(\theta_{1}, \ldots, \theta_{n}\right)$ be an integral interior angle data and $m \geq 3$ be a number such that $D(\Theta, m)$ is not empty. We are interested in the flat metrics on $\overline{\mathbb{H}}$ which are of the form $\mu(A,(\hat{z}, \hat{x}))$, where $(\Theta, A)$ is a good pair and $(\hat{z}, \hat{x}) \in X(n, m)$. $\overline{\mathbb{H}}$ together with the metric $\mu(A, \hat{z}, \hat{x})$ will be denoted by $\overline{\mathbb{H}}(A,(\hat{z}, \hat{x}))$. Therefore $U(\overline{\mathbb{H}}(A,(\hat{z}, \hat{x}))$ is the unit tangent bundle of $\overline{\mathbb{H}}(A,(\hat{z}, \hat{x}))$.

Since $\Theta$ is integral, a flat disk having interior angle data $\Theta$ has trivial holonomy group. In particular, the parallel transport of a vector does not depend on the path chosen. For each $\overline{\mathbb{H}}(A,(\hat{z}, \hat{x}))$, consider the directed edge joining the vertex $x_{1}$ to the $x_{j}$, where $x_{j}$ is the vertex after $x_{1}$ with respect to the cyclic order on the boundary of $\overline{\mathbb{H}}$. It is clear that $j$ is constant on a connected component of $X(n, m)$. To each $(y, \alpha) \in \overline{\mathbb{H}} \times \mathbb{R} / 2 \pi \mathbb{Z}$, we associate the vector in $U(\overline{\mathbb{H}}(A,(\hat{z}, \hat{x}))$ which is based at $y$ and has parallel transport equal to the vector making an angle of $\alpha$ with the directed edge that we chose above. This implies that

$$
U(\overline{\mathbb{H}}(A,(\hat{z}, \hat{x}))=\overline{\mathbb{H}} \times \mathbb{R} / 2 \pi \mathbb{Z}
$$

Theorem 3 Let $\Theta$ be an integral angle data and $m \geq 3$ be an integer such that $D(\Theta, m)$ is not empty. There exists a dense $G_{\delta}$ subset in $D(\Theta, m)$ such that for each element in this set, the billiard flow is topologically transitive.

Proof. We identify $D(\Theta, m)$ with $E(\Theta) \times X(n, m)$, and for each $(A,(\hat{z}, \hat{x})) \in E(\Theta) \times X(n, m)$ we consider the flat disk $\overline{\mathbb{H}}(A,(\hat{z}, \hat{x}))$. Since $E(\Theta) \times X(n, m)$ is a manifold, we can put a complete metric on it. 
e-ISSN: 2458-7575 (http://dergipark.gov.tr/bseufbd)

For $\quad$ each $\quad(A,(\hat{z}, \hat{x})) \in E(\Theta) \times X(n, m), \quad$ consider $\quad$ the $\quad$ following $\quad$ metric $\quad$ on
$U(\mathbb{H}(A,(\hat{z}, \hat{x}))=\overline{\mathbb{H}} \times \mathbb{R} / 2 \pi \mathbb{Z}:$

$$
d_{(A, \hat{z}, \hat{x})}\left((y, \alpha),\left(y^{\prime}, \alpha^{\prime}\right)\right)=\max \left\{\mu_{(A, \hat{z}, \hat{x})}\left(y, y^{\prime}\right), d_{0}\left(\alpha, \alpha^{\prime}\right)\right\}
$$

where $d_{0}\left(\alpha, \alpha^{\prime}\right)$ is the distance between $\alpha$ and $\alpha^{\prime}$ in $\mathbb{R} / 2 \pi \mathbb{Z}$.

Note that the topologies induced by $(A,(\hat{z}, \hat{x}))$ and $\left(\left(A^{\prime},\left(\hat{z}^{\prime}, \hat{x}^{\prime}\right)\right)\right.$ on $\overline{\mathbb{H}} \times \mathbb{R} / 2 \pi \mathbb{Z}$ are same. Let $\left\{a_{1}, a_{2}, a_{3}, \ldots\right\}$ be a dense subset of $\overline{\mathbb{H}} \times \mathbb{R} / 2 \pi \mathbb{Z}$ and $\left(r_{k}\right)_{k=1}^{\infty}$ be a sequence of positive numbers which converges to 0 . Let $B_{A,(\hat{z}, \hat{x})}(a, r)$ denote the ball in $\overline{\mathbb{H}} \times \mathbb{R} / 2 \pi \mathbb{Z}$ with radius $r$ and center $a$ with respect to the metric (8). Then the $\operatorname{set}\left\{B_{A, \hat{z}, \hat{x}}\left(a_{i}, r_{k}\right): i, k \in \mathbb{N}\right\}$ form a basis for $\overline{\mathbb{H}} \times \mathbb{R} / \mathbb{Z}$ for each $A,(\hat{z}, \hat{x})$.

Let $A_{l}$ be the set of $(A,(\hat{z}, \hat{x})) \in E(\Theta)$ such that for each open set $U$ in the phase space, there exists a billiard trajectory starting in $U$ which visits $B_{(A, \hat{z}, \hat{x})}\left(a_{i}, r_{k}\right)$ for each $i, k \leq l$, in the phase space of the billiard flow in $\mathbb{H}(A,(\hat{z}, \hat{x}))$. Each $A_{l}$ is open and $\cap_{l=1}^{\infty} A_{l}$ is a $G_{\delta}$ set.

We show that this intersection is dense. Let $Y_{s}$ be the space of all $(A,(\hat{z}, \hat{x})) \in E(\Theta) \times X(n, m)$ such that $A=\left(\frac{k_{1}}{l_{1}} \pi, \ldots \frac{k_{m}}{l_{m}} \pi\right), k_{i}, l_{i}$ are coprime, and the least common multiple of $l_{i}$ 's is greater than $s$. For each element $A_{s}$, the corresponding flat disk is really flat. Therefore for each $(A,(\hat{z}, \hat{x})) \in A_{s}$, we can consider the canonical cover $d^{*}(\overline{\mathbb{H}}(A,(\hat{z}, \hat{x})))$ of the doubling of $\overline{\mathbb{H}}(A,(\hat{z}, \hat{x}))$. Since it is very flat, each trajectory of a geodesic is dense except for countably many directions. Such a trajectory induces an orbit of the flow on $\mathbb{H} \times \mathbb{R} / 2 \pi \mathbb{Z}$, and closure of this orbit is at most in a $\frac{\pi}{s}$ distance from any point of the phase space, since the degree of the canonical cover is greater than or equal to $s$. Therefore for each $l$, there exists $N$ such that for all $s \geq N$ we have $Y_{s} \subset A_{l} . Y_{s}$ is dense in $E \times X(n, m)$, hence $A_{l}$ is dense also. Therefore Baire's Category Theorem implies that $\bigcap A_{l}$ is dense.

Now fix $(A,(\hat{z}, \hat{x})) \in \cap A_{l}$. We show that the flow on $\overline{\mathbb{H}} \times \mathbb{R} / 2 \pi \mathbb{Z}$ has a dense orbit. Let $\bar{B}_{1}$ be a closed ball in $\overline{\mathbb{H}} \times \mathbb{R} / 2 \pi \mathbb{Z}$ such that for each element in this ball the corresponding orbit intersects with $B_{A, \hat{z}, \hat{x}}\left(a_{1}, r_{1}\right)$. For each $l \geq 2$, let $\bar{B}_{r}$ be a closed ball such that for each element in this ball corresponding orbit intersects $B_{A, \hat{z}, \hat{x}}\left(a_{i}, r_{k}\right)$, for all $i, k \leq l . \cap B_{l}$ is not empty. For each element $(y, \alpha)$ in this set, the orbit of the billiard flow which starts at $y$ and has direction $\alpha$ is dense in the phase space.

\section{REFERENCES}

[1] Gutkin, E. (1996). Billiards in polygons: survey of recent results. Journal of statistical physics, 83(1), 7-26.

[2] Gutkin, E. (2003). Billiard dynamics: a survey with the emphasis on open problems. Regular and chaotic dynamics, 8(1), 1-13.

[3] Masur, H. (2006). Ergodic theory of translation surfaces. Handbook of dynamical systems 1B. Elsevier, 527-547.

[4] Zorich, A. (2006). Frontiers in number theory, physics, and geometry I, APA, 439-585

[5] Masur, H. \& Tabachnikov, S. (2002). Rational billiards and flat structures. Handbook of dynamical systems 1A. Elsevier, 1015-1089.

[6] Sağlam, İ. (2016). Flat Surfaces with Finite Holonomy Group. arXiv preprint arXiv:1612.07169. 
[7] Zemlyakov, A. N., \& Katok, A. B. (1975). Topological transitivity of billiards in polygons. Mathematical Notes of the Academy of Sciences of the USSR, 18(2), 760-764.

[8] Troyanov, M. (2007). On the Moduli Space of Singular Euclidean Surfaces. Handbook of Teichmüller Theory 1, AMS, 507-540.

[9] Troyanov, M. (1986). Les surfaces euclidienne à singularités coniques. L'Enseignement Math. EMS, 79-94.

[10] Troyanov, M. (1991). Prescribing curvature on compact surfaces with conical singularities. Transactions of the American Mathematical Society, 324(2), 793-821.

[11] Hulin, D.,\& Troyanov, M. (1992). Prescribing curvature on open surfaces. Mathematische Annalen, 293(1), 277-315.

[12] Sağlam, İ. (2016). Complete Flat Cone Metrics on Surfaces. arXiv preprint arXiv:1602.04240 